\title{
Antigenic Polymorphism of Human Very Late Activation Protein-2 (Platelet Glycoprotein la-lla)

\author{
Platelet Alloantigen $\mathrm{Hc}^{\mathrm{a}}$
}

Virgil L. Woods, Jr., Ken D. Pischel, Esther D. Avery, and Harry G. Bluestein

Division of Rheumatology, Department of Medicine, University of California Medical Center, San Diego, California 92103

\begin{abstract}
We have found evidence for a human alloantigenic system on the very late activation protein -2 (VLA-2) heterodimer (platelet GPIa/IIa). Sera from two patients with systemic lupus erythematosus (SLE) contained antibodies that immunoprecipitated surface molecules from platelets and fibroblasts that comigrated on SDS-PAGE and two-dimensional O'Farrell gels with platelet GPIa (VLA-alpha 2 chain) and platelet GPIIa (VLA-beta chain). These SLE antibodies were alloreactive as they precipitated VLA molecules from only 5 of 22 normal donors' platelets and did not react with the lupus patients' own platelets, despite the expression of apparently normal amounts of VLA on the donors' cells. Two-dimensional $O$ 'Farrell analysis demonstrated no differences in the molecular weight or isoelectric point of GPIa and GPIIa obtained from platelets of alloantibody reactive or unreactive donors. Sequential immunoprecipitation experiments with VLA chainspecific monoclonal antibodies, and the pattern of immunoprecipitation of several different VLA heterodimers demonstrated that the alloantibody-reactive determinant was present on the VLA-2 heterodimer, and not other VLA molecules. Thus, these SLE sera demonstrate a previously unrecognized antigenic polymorphism of the VLA-2 (platelet GPIa/IIa) heterodimer, platelet alloantigen $\mathrm{Hc}^{\text {n. }}$
\end{abstract}

\section{Introduction}

Very late activation proteins (VLA) ${ }^{1}$ are a family of heterodimeric cell surface glycoproteins originally identified on activated human $\mathrm{T}$ lymphocytes. They were subsequently shown to be present on a wide variety of cell types including fibroblasts and platelets. Six forms (VLA-1 to VLA-6) have been identified, each consisting of a distinct alpha chain (alpha $a_{1}$ to alpha $_{6}$ associated with a common beta chain (1-6). The VLA proteins are members of a superfamily of structurally and functionally related adhesion-mediating cell surface proteins that include the human fibroblast fibronectin receptor (VLA-5), the LFA-1/Mac-1 group, and the platelet GPIIb/ IIIa-vitronectin receptor group (7-10). We have recently

Address reprint requests to Dr. Woods, University of California, San Diego, 225 Dickinson Street, H-81 1-G, San Diego, CA 92103.

Received for publication 22 February 1988 and in revised form 5 October 1988.

1. Abbreviations used in this paper: ACD, acid citrate dextrose; GP, platelet glycoprotein; TBS, Tris-buffered saline; VLA, very late activation antigen.

J. Clin. Invest.

(c) The American Society for Clinical Investigation, Inc.

0021-9738/89/03/0978/08 \$2.00

Volume 83, March 1989, 978-985 shown that VLA proteins on platelets are indistinguishable from platelet membrane glycoproteins (GP) Ia, IIa, and Ic, and exist as a mixture of GPIa-IIa (VLA-2) and GPIc-IIa heterodimers $(11,12)$.

We have reported that VLA proteins are exceptionally immunogenic when used as heteroimmunogens (13). If human allotypic forms of these molecules exist, such polymorphisms might account for some of the less well characterized alloantigenic systems known to be present on human platelets (14), and alloantibodies directed at these variants might play a pathogenic role in situations where alloimmunization can occur. In this report, we present evidence for alloantigenic forms of human VLA proteins. Sera from two systemic lupus erythematosus (SLE) patients who had previously received multiple blood transfusions have been found to contain alloantibodies that react with a commonly occurring (23\% of normal donors) allotypic determinant found on the VLA-2 heterodimer (platelet GPIa/IIa).

\section{Methods}

Cell preparations. $30 \mathrm{ml}$ of venous blood was obtained from healthy adults and SLE patients, mixed with $5 \mathrm{ml}$ acid-citrate dextrose $(13.5 \mathrm{~g}$ citric acid, $25 \mathrm{~g} \mathrm{Na}$ citrate, $20 \mathrm{~g}$ dextrose per liter, $\mathrm{pH} 4.5, \mathrm{ACD})$ and centrifuged at $180 \mathrm{~g}$ for $15 \mathrm{~min}$ at $22^{\circ} \mathrm{C}$ to yield platelet-rich plasma (15). The platelets were then washed twice by suspension and centrifugation in $0.15 \mathrm{M} \mathrm{NaCl}, 0.01 \mathrm{M}$ Tris (Tris-buffered-saline, TBS) pH 6.5 at $1,000 \mathrm{~g}$ for $15 \mathrm{~min}$ and then radioiodinated. Preparations of peripheral blood mononuclear cells (PBMs) were obtained by Ficoll-Hypaque (Pharmacia Fine Chemicals, Piscataway, NJ) density gradient centrifugation of heparin-anticoagulated whole blood and washed three times with PBS. The PBMs obtained in this manner contained large amounts of platelets (two to four platelets per mononuclear cell). Fibroblast lines were isolated from skin biopsies of selected donors and maintained in monolayer culture (16).

Radiolabeling of cell surface proteins. Washed platelets $\left(1 \times 10^{9}\right)$, PBM-platelets $\left(5 \times 10^{7}\right.$ PBM plus $1-2 \times 10^{8}$ platelets) or fibroblasts $(5$ $\times 10^{7}$ ) were suspended in $500 \mu \mathrm{l} 0.15 \mathrm{M} \mathrm{NaCl}, 0.01 \mathrm{M} \mathrm{PO}_{4}, \mathrm{pH} 7.4$ (PBS) or TBS pH 7.4, and reacted with $1 \mathrm{mCi} \mathrm{Na}{ }^{125} \mathrm{I}$ (New England Nuclear, North Billerica, MA), $10 \mu$ lactoperoxidase (CalbiochemBehring Corp., San Diego, CA; B grade, $100 \mathrm{IU} / \mathrm{ml}$ ) and $25 \mu \mathrm{l}$ of $0.03 \%$ $\mathrm{H}_{2} \mathrm{O}_{2}$. After $5 \mathrm{~min} 5 \mu \mathrm{l}$ of lactoperoxidase and $10 \mu \mathrm{l}$ of $0.03 \% \mathrm{H}_{2} \mathrm{O}_{2}$ were added, and at $10 \mathrm{~min} 10 \mu \mathrm{l}$ of $0.03 \% \mathrm{H}_{2} \mathrm{O}_{2}$ was added. After 15 min the reaction was stopped by the addition of cold PBS (PBM-platelets, fibroblasts) or TBS pH 6.5 (platelets), cells pelleted by centrifugation, and washed three additional times $(15,17)$.

$S L E$ patients with alloantibody. Serum and plasma were obtained from patient H.C., a 60-yr-old G3, P3, AbO Mexican-American female diagnosed as having SLE in 1980 with malar rash, arthralgias, lymphadenopathy, fever, a positive lupus band test, a strongly positive antinuclear antibody test and biopsy-documented glomerulonephritis. She has received corticosteroid therapy (prednisone, $\sim 20 \mathrm{mg}$ daily) from 1980 to the present. In 1980 she received a transfusion of $2 U$ of packed red blood cells at the time of a nephrectomy necessitated by pyelonephritis secondary to stone-induced ureteric obstruction. At the 
time of E35 plasma aquisition in 1986, the patient had a creatinine of 1.2 and mild proteinuria. She has not had SLE CNS disease, hemolytic anemia, leukopenia, or thrombocytopenia. At the time her serum was obtained for these studies her platelet count was $232,000 / \mathrm{cm}^{3}$. The patient has never had episodes of unusual bleeding, or vascular thrombosis. The patient had her bleeding time determined on one occasion (bleeding time of $3 \mathrm{~min}, 8 / 24 / 84$ ). The patient's prothrombin time has been normal throughout her illness while her partial thromboplastin time has consistently been shortened (23.4 with a control of 26.7), presumably due to her chronic corticosteroid usage.

Serum E36 was obtained from SLE patient G.F., a 62-yr-old G18, P5, Ab13 woman with a history of poorly controlled hypertension, who experienced rapid worsening of chronic renal failure, for which she was hospitalized on 6/20/86. Evaluation revealed nephrotic proteinuria and biopsy-documented glomerulonephritis with focal sclerosis and crescent formation consistent with lupus nephritis. At that time the patient had a positive antinuclear antibody (titer of $1: 120$ ) with negative double-stranded DNA antibodies. In retrospect, it was found that the patient had a several year history of photosensitive skin rash, recurrent arthralgias, pericarditis, recurrent miscarriages, usually in the first trimester of pregnancy, and a positive serologic test for syphilis for which she had been treated in the past with penicillin. The patient had no history of bleeding or thrombotic episodes, and had not experienced documented SLE CNS disease, though she had a history of major depression with multiple suicide attempts dating to 1971 . The serum sample (E36) for our studies was drawn $6 / 23 / 86$. While hospitalized, her platelet count ranged between 205,000 and $188,000 / \mu l^{3}$, and her bleeding time was $7 \mathrm{~min}$, prothrombin time was 11.9 with a control of 11.4, and her partial thromboplastin time was 23 with a control of 30 . The patient was treated with intravenous bolus cytoxan and corticosteroids, but she developed further renal failure, bacterial sepsis, and died 1 mo later.

Antibodies. Sera and whole blood for cell isolation were obtained from SLE patients followed at the UCSD Medical Center Rheumatology Clinic who met the 1982 American Rheumatism Association Criteria for SLE, and from healthy laboratory personnel or paid donors. All serum samples were heat inactivated at $56^{\circ} \mathrm{C}$ for $30 \mathrm{~min}$ and stored at $-80^{\circ} \mathrm{C}$.

Monoclonal antibodies (MAb) 12F1 and A-1A5 are VLA chainspecific monoclonal antibodies that we have been previously described $(1,12,18)$. MAb $12 \mathrm{~F} 1$ is reactive with an epitope on the VLA-alpha ${ }_{2}$ heavy chain (platelet GPIa) and immunoprecipitates only the VLA-2 heterodimer (platelet GPIa-IIa) from detergent extracts of platelets or fibroblasts. A-1A5 (generous gift of Martin Hemler, Dana Farber Cancer Institute) is reactive with an epitope on the beta chain common to all VLA heterodimers and immunoprecipitates both VLA-2 (GPIaIIa) and GPIc-Ila heterodimers from platelet extracts. A-1A5 immunoprecipitates these, and additional VLA heterodimers from fibroblast extracts. MAb 4.22 is a control monoclonal antibody directed at mouse IgD (15).

Extraction and immunoprecipitation. Surface radioiodinated cells were incubated with human serum or MAb, washed, solubilized in nonionic detergent, and platelet-antigen-antibody complexes immunoprecipitated with Sepharose-bound protein-A as previously described $(15,17)$. In some experiments, the platelets contained in Ficoll-Hypaque purified PBM preparations were used to characterize platelet VLA antigens. We have previously shown that the VLA-alpha chain in such preparations originate exclusively from the platelets (18). Briefly, $100 \mu \mathrm{l}$ of serum or $1 \mu \mathrm{g}$ of MAb was incubated with intact cells $\left(1 \times 10^{8}\right.$ platelets, $5 \times 10^{6}$ PBM-platelets, $1-2 \times 10^{6}$ fibroblasts $)$ for 30 min at $4^{\circ} \mathrm{C}$ and the cells washed with TBS or PBS pH 7.4. Platelets were solubilized $\left(1 \times 10^{9} / \mathrm{ml}\right.$ final concentration) by incubation for 30 min at $4^{\circ} \mathrm{C}$ in TBS pH 7.4 containing $1 \%$ Triton $\mathrm{X}-100$, and protease inhibitors $(0.06 \mathrm{mM} N$-a-p-tosyl-L-lysine choromethyl ketone [TLCK], $0.06 \mathrm{mM} \mathrm{L-1-Tosylamide-2-phenylethyl} \mathrm{chloromethyl} \mathrm{ke-}$ tone [TPCK], and $0.2 \mathrm{mM}$ PMSF, all obtained from Sigma Chemical Co., St. Louis, MO). PBM-platelets $\left(2 \times 10^{7} / \mathrm{ml}\right)$ or fibroblasts $(1$ $\times 10^{6} / \mathrm{ml}$ ) were solubilized in TBS pH 7.4, $0.5 \%$ NP-40 (TBS-NP-40) containing the above protease inhibitors. Cellular lysates were then ultracentrifuged at $100,000 \mathrm{~g}$ for $30 \mathrm{~min}$ at $4^{\circ} \mathrm{C}$ and used immediately or frozen at $-70^{\circ} \mathrm{C}$ until used.

Aliquots were precleared by incubation with $50 \mu$ l of Sepharose 4B for 15 min followed by centrifugation (microfuge B, Beckman Instruments, Inc., Palo Alto, CA) for $4 \mathrm{~min}$. The immune complexes in the extracts were collected by incubation $\left(1 \mathrm{~h} 4^{\circ} \mathrm{C}\right)$ with $10 \mu$ l SepharoseProtein A (Pharmacia Fine Chemicals, $1 \mathrm{mg}$ protein $\mathrm{A} / \mathrm{ml}$ ), or with 10 $\mu l$ of affinity purified rabbit antimouse immunoglobulin kappa chain conjugated to Sepharose 4B $(1 \mathrm{mg} / \mathrm{ml})$ according to the manufacturer's instructions. Sepharose conjugates were then pelleted by centrifugation, washed once with TBS-NP-40, layered onto $1 \mathrm{ml}$ of $20 \%$ sucroseTBS-NP-40, and then pelleted by centrifugation.

$S D S-P A G E$. Washed immunoprecipitates were eluted and denatured with $25 \mu \mathrm{l} 1 \%$ SDS and 5\% 2-mercaptoethanol (2-ME) by heating at $100^{\circ} \mathrm{C}$ for $2 \mathrm{~min}$ and analyzed along with molecular weight markers by 7 or $10 \%$ Laemmli SDS polyacrylamide gel electrophoresis (SDSPAGE) as previously described (17). Gels were fixed in $10 \%$ acetic acid, $10 \%$ 2-isopropyl alcohol then dried and autoradiographed with prefogged XAR-5 film (Eastman Kodak, Rochester, NY) using an intensifying screen (Cronex Lighting-Plus; E.I. Dupont de Nemours, Wilmington, DE) for $1-7 \mathrm{~d}$ at $-80^{\circ} \mathrm{C}$.

Sequential immunoprecipitation. Nonionic detergent extracts of alloantibody containing SLE sera E35 or E36 or control serum preincubated platelets were prepared as above, and aliquots incubated $(1 \mathrm{~h}$ $\left.4^{\circ} \mathrm{C}\right)$ with MAb bound to Sepharose $4 B(50 \mu 1,1 \mathrm{mg} / \mathrm{ml})$, either A-1A5, $12 \mathrm{~F} 1$ or control MAb 4.22. The resulting extracts were tested for their residual content of $\mathrm{MAb}$-reactive material by further immunoprecipitation with MAb A-1A5 Sepharose or MAb 12F1 Sepharose and subsequent SDS-PAGE/autoradiography as described above. This procedure was repeated until material reactive with the preclearing MAb Sepharose was no longer detectable in the extract (usually five sequential preclearances). The resulting extract was then reacted either with Protein-A Sepharose $(10 \mu \mathrm{l})$, to precipitate human alloantibody-antigen complexes, or with the MAb-Sepharose conjugates, and the precipitated platelet proteins analyzed by SDS-PAGE/autoradiography.

The experiment was also performed in the reverse order, with extracts first being cleared of alloantibody-antigen complexes with Protein-A Sepharose, and then the resulting extracts tested for their residual content of VLA molecules.

O'Farrell two-dimensional gel electrophoresis (2D-PAGE). Twodimensional PAGE was done by the method of O'Farrell as previously described (17). Samples were eluted from the Sepharose immunoadsorbents by incubation with $8 \mathrm{M}$ urea, $5 \% 2-\mathrm{ME}$ for $60 \mathrm{~min}$ at $22^{\circ} \mathrm{C}$ and applied to prefocused $6 \%$ acrylamide tube gels with $10 \mathrm{mM}$ phosphoric acid ( $\mathrm{pH} \mathrm{3.9)}$ and $50 \mathrm{mM}$ Tris base ( $\mathrm{pH} \mathrm{9.9)} \mathrm{electrode} \mathrm{solutions,}$ and focused for $8 \mathrm{~h}$ at $1,000 \mathrm{~V}$. The gels were then equilibrated with SDS-PAGE diluent buffer, run on reduced 7\% SDS-PAGE in the second dimension and fixed and processed as described above for 1DSDS-PAGE.

Platelet-eluted alloantibodies. Platelet eluates enriched for alloantibody were prepared by a procedure similar to that previously employed to purify alloantibodies to platelet GPIIb-IIIa (19). Washed platelets, obtained from both an alloantibody-reactive and unreactive donor $\left(1 \times 10^{9}\right.$ platelets $)$ were incubated with $10 \mathrm{ml}$ of alloantibodycontaining plasma E35 for $2 \mathrm{~h}$ at $4^{\circ} \mathrm{C}$ in the presence of $1 \mu \mathrm{g} / \mathrm{ml}$ prostaglandin $E_{1}$, and $0.18 \mathrm{mg} / \mathrm{ml}$ theophyllin, both obtained from Sigma Chemical Co. Platelets were then pelleted by centrifugation at $1,000 \mathrm{~g}$ for $15 \mathrm{~min}$, washed twice in TBS, and adsorbed antibodies were eluted by the slow addition (over $5 \mathrm{~min}$ ) of sufficient $0.1 \mathrm{M} \mathrm{HCl}$ to bring the $\mathrm{pH}$ of the platelet suspension to 3.0. Platelets were then pelleted at $10,000 \mathrm{~g}$, the supernatant collected and brought to $\mathrm{pH} 7.4$ by the addition of $1.0 \mathrm{M}$ Tris- $\mathrm{HCl}, \mathrm{pH} 8.0$, and then dialyzed overnight at $4^{\circ} \mathrm{C}$ against TBS, pH 7.4. The eluate prepared from alloantigen-positive platelets strongly immunoprecipitated both GPIa and GPIIa and precipitated no other platelet molecules.

Immunoblot studies with serum E35. Washed platelets obtained from an SLE E35-reactive and an SLE E36-unreactive donor were 
solubilized in TBS-NP-40 containing protease inhibitors, ultracentrifuged, and then immunoprecipitated with VLA-2 specific monoclonal antibody $12 \mathrm{~F} 1$, or VLA beta chain-specific monoclonal antibody A-1A5, followed by collection of immunoprecipitates with Protein-A Sepharose. After washing, the immunoprecipitates were subjected to 7.5\% SDS-PAGE, both reduced with $0.5 \% 2-\mathrm{ME}$ and unreduced. The SDS-PAGE-separated glycoproteins were then electrophoretically transferred to nitrocellulose sheets by the technique of Western blot. Nitrocellulose sheets bearing transferred platelet proteins were first blocked by a 1-h incubation with PBS, pH 7.4, containing 5\% skim milk, and $0.05 \%$ Tween 20 . The blocked nitrocellulose was then cut into strips, and individual strips incubated overnight with 1:50 dilutions of alloantibody-containing serum E35, alloantibody negative SLE sera, and 1:2 dilutions of platelet eluate (see above) containing purified and concentrated alloantibody from E35 sera. The strips were then washed in TBS- $0.05 \%$ Tween and incubated with biotinylated, affinity-purified goat $\mathrm{Fab}_{2}$ antibody specific for human IgG (diluted 1:500; Caltag Laboratories, San Francisco, CA). After further washing, the strips were incubated with high molecular weight avidin-biotinylated peroxidase complexes (ABC; Vector Scientific, San Diego, CA), washed, and incubated with ELISA substrate $(6.0 \mathrm{mg}$ 4-chloro 1naphthol, $10 \mu \mathrm{l} 30 \% \mathrm{H}_{2} \mathrm{O}_{2}$ in $10.0 \mathrm{ml}$ PBS, pH 7.4).

Platelet aggregation studies. Platelet-rich plasma was prepared from anticoagulated whole blood (one part $0.04 \mathrm{M}$ citric acid, $0.06 \mathrm{M}$ sodium citrate plus 9 parts blood) obtained from both an allotype positive and an allotype negative aspirin-free normal donors. Plateletrich plasma $(400 \mu \mathrm{l})$ from each of the donors was then mixed with $50 \mu \mathrm{l}$ of test plasma, either patient E35 (containing alloantibody, control SLE plasma (known not to contain alloantibodies), or the platelet donors' own platelet poor plasma, or $50 \mu \mathrm{l}$ of platelet eluate containing concentrated alloantibody, and then incubated for $15 \mathrm{~min}$ at room temperature without stirring. The platelet suspensions were then stirred and warmed to $37^{\circ} \mathrm{C}$ in a platelet aggregometer (PAP-4 Platelet Aggregation Profiler, Bio/Data, Hatboro, PA). Platelet suspensions were then activated by the addition $(50 \mu \mathrm{l})$ of stock solutions of collagen fibrils obtained from calf skin (final concentration $0.2 \mathrm{mg} / \mathrm{ml}$, catalogue 885-1), ADP (final concentration of $5 \mu \mathrm{M}$ or ristocetin (final concentration $1.2 \mathrm{mg} / \mathrm{ml}$ ), all obtained from Sigma Chemical Co.

\section{Results}

Antibodies that immunoprecipitate cell surface molecules of $\sim 160$ and $135 \mathrm{kD}$ on reduced and unreduced SDS-PAGE were detected in 2 of 38 SLE sera. Those two sera, E35 and E36, immunoprecipitated glycoproteins that comigrated on one-dimensional SDS-PAGE with molecules precipitated by the VLA specific monoclonal antibody A-1A5 (Fig. 1, $A$ ). These membrane molecules were not immunoprecipitated by sera obtained from a group of 21 healthy human donors (data not shown).

The molecules immunoprecipitated from platelet membranes by SLE sera and by the VLA-beta chain specific monoclonal A-1A5 were compared on two-dimensional O'Farrell gels (Fig. 2). SLE sera and A-1A5 precipitated the same pair of glycoproteins (one of $160 \mathrm{kD}$ with a pI of 5.0 and 1 of $135 \mathrm{kD}$ with pI 4.5). These molecules correspond to the VLA-alpha and VLA-beta chains previously shown to be identical to platelet GPIa and GPIIa. A-1A5 also immunoprecipitated a molecule of $130-135 \mathrm{kD}$ with a pI of 4.9 , which has been identified as platelet GPIc-alpha. The absence of that molecule in the SLE immunoprecipitate indicates that the lupus antibodies react only with the VLA-2 heterodimer.

Sera E35 and E36 reacted with VLA molecules on cell membranes from some but not all normal donors. For example, they did not immunoprecipitate either the 160 or $135 \mathrm{kD}$ glycoproteins from platelets obtained from donor E.L. (Fig. 1, $B$ ) although those cells were richly endowed with VLA molecules that were immunoprecipitated with monoclonal antibody A-1A5. When tested against platelets from a group of normal donors, E35 immunoprecipitated the 160 - and 135-kD molecules from 5 of 17 donors' platelets while E36 immunoprecipitated them from 5 of 22 donors' platelets. E35 and E36 reacted concordantly with the same five donors. In additional experiments, we immunopurified IgG from E35 serum with the use of Protein A Sepharose and then used the purified IgG to immunoprecipitate platelet extracts. Purified E35 IgG precipitated VLA molecules from platelets previously found to be reactive with E35 serum. This result established that the alloantibody as a purified IgG could bind to VLA antigens without the participation of serum or plasma cofactors.

Serum E35 was also tested for its ability to react with platelets obtained from the SLE patient who donated that serum. E35 did not immunoprecipitate the 160- and 135-kD molecules, but monoclonal antibody A-1A5 precipitated normal amounts of the VLA glycoproteins from those cells. Thus, the anti-VLA reactivities in those SLE sera are alloreactive and are not autoantibodies.

In an attempt to define the structural basis for the alloantigenic differences observed between VLA molecules on SLE antibody-reactive and unreactive platelets, both types of platelets were immunoprecipitated with monoclonal A-1A5 and the mobilities of VLA molecules compared on two-dimensional O'Farrell gels. No differences in molecular weight or isoelectric point of the VLA molecules were seen; thus, the allotypic variation is due to a fairly limited structural change.

We examined the reactivity of SLE sera E35 and E36 with VLA molecules on cultured skin fibroblasts and compared these immunoprecipitates with those from the same donor's platelets. Representative results, obtained with cells from donor R.W., are shown in Fig. 3. SDS-PAGE analysis of the immunoprecipitates from both cell types produced by sera E35 and E36 revealed 2 bands at 160 and $135 \mathrm{kD}$ (panels $A$ and $B$ ). Thus, the alloreactive epitope is not restricted to platelets. The fibroblast immunoprecipitates obtained with VLA-2 specific monoclonal antibody $12 \mathrm{~F} 1$ contained two bands of the same apparent molecular weights as those produced by E35 and E36 $(B)$. Fibroblasts are a rich source of several different VLA glycoproteins as shown by the A-1A5 immunoprecipitates. Since E35 and E36 appear to precipitate only the VLA-2 heterodimer from fibroblasts, the alloreactivity to VLA contained in these sera appear to be directed at an epitope unique to VLA-2. If reactivities were directed at beta chain epitopes present on other VLA heterodimers then additional VLA heavy chains would have been expected in the fibroblast precipitates. We have studied the reactivity of E35 and E36 with fibroblast lines obtained from five normal donors. Three of these donors' platelets were unreactive with either E35 or E36, and their fibroblasts were also found to be unreactive with E35 and E36. Two of the donors had platelets reactive with both sera, and VLA-2 was strongly precipitated from their fibroblasts by both E35 and E36. Therefore, there is complete concordance in the reactivity of the two antibodies towards different cell types of individual donors.

The reactivity of the SLE alloantibodies with an epitope unique to the VLA-2 heterodimer was confirmed by sequential immunoprecipitation experiments. Platelets from donor E.A. were incubated with E35 or normal human serum (NHS), 


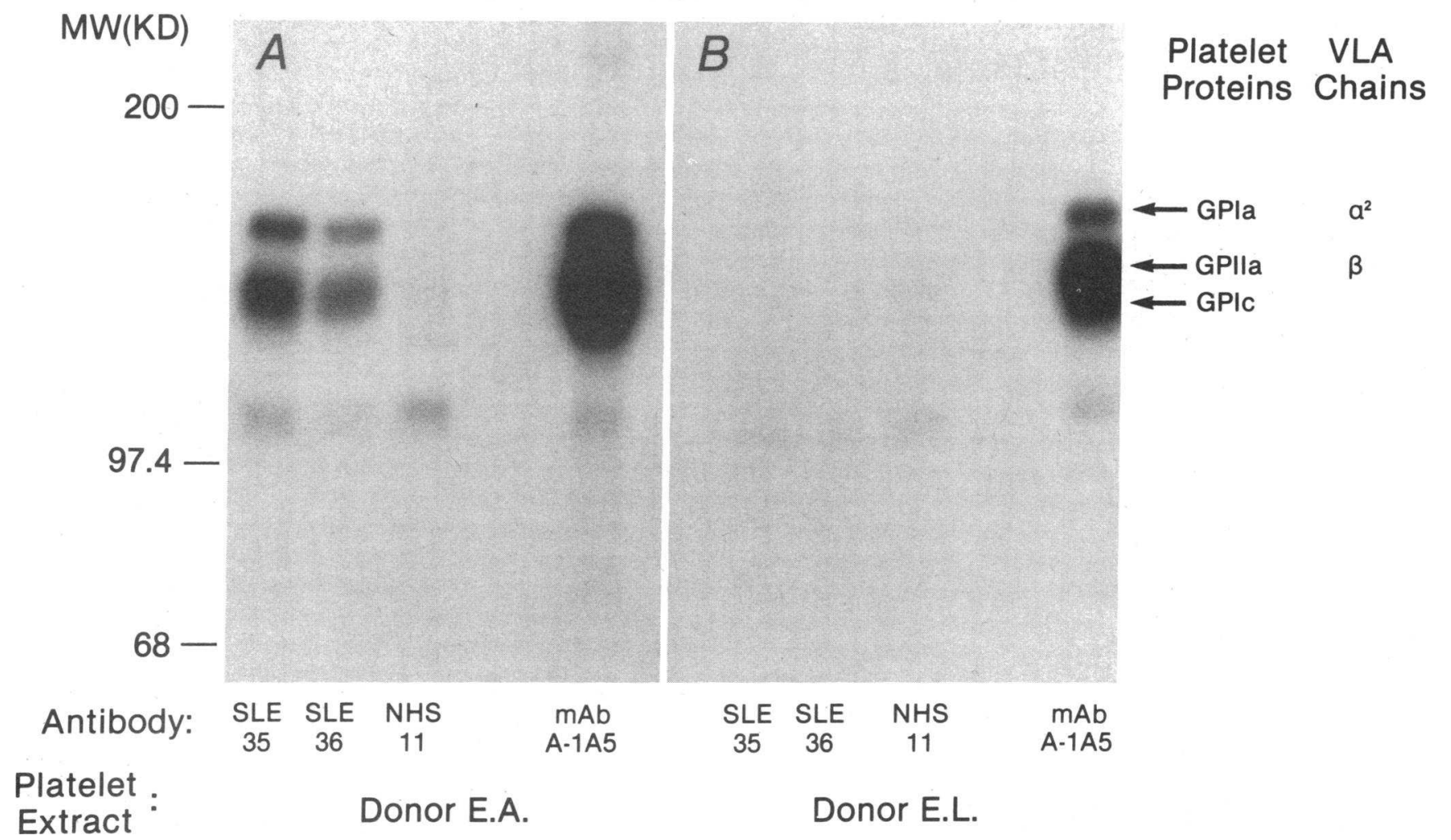

Figure 1. Immunoprecipitation of platelet surface molecules by SLE sera E35, E36 and by VLA-specific monoclonal antibody A-1A5. Platelets from normal donors E.L. and E.A., were radioiodinated and then incubated with SLE or normal human sera, or monoclonal antibody A-1A5. The antibody-incubated platelets were then washed, solubilized in 1\% Triton X-100-TBS, antibody-bound platelet molecules isolated with Protein A Sepharose, and analyzed along with molecular weight markers on 10\% Laemmli SDS polyacrylamide gels, followed by processing for autoradiography. SLE sera E35 and E36 immunoprecipitated material from donor E.A.'s platelets, which co-migrated with molecules immunoprecipitated from the same platelets by the VLA specific monoclonal A-1A5 $(A)$. However, the same sera failed to immunoprecipitate material from donor E.L.'s platelets, even though comparable amounts of VLA-specific MAb reactive material were present on these platelets $(B)$. The bands at approximately $110 \mathrm{kD}$ represent nonspecifically precipitated material.

washed, and nonionic detergent extracts were prepared. The extracts were precleared of VLA molecules by incubation with either A-1 A5-Sepharose, 12F1-Sepharose or control MAb 4.22-Sepharose, and then tested for their residual content of SLE E35 alloantibody-reactive VLA molecules (Fig. 4). Preclearance with either monoclonal antibody A-1A5 or $12 \mathrm{~F} 1$ removed SLE E35-reactive VLA molecules from the extract. This result confirmed the specificity of these alloantibodies for VLA molecules. It further demonstrated that removal of platelet VLA-2 by the alpha-2 chain-specific monoclonal $12 \mathrm{~F} 1$ was sufficient to remove all SLE E35-reactive material from the extract even though other VLA heterodimers remained in the extracts, as evidenced by their reactivity with the anti-beta chain monoclonal A-1A5 (Fig. 4 b). Thus, the E35 reactivity is directed at unique features of the VLA-2 heterodimer and not to beta chain epitopes common to all platelet VLA heterodimers.

The sequential clearances were also performed in the reverse order; extracts first cleared of alloantibody-antigen complexes with Protein A Sepharose, and then tested for their residual content of VLA molecules. Platelet extracts depleted of E35 alloantibody-antigen complexes were $\sim 50 \%$ depleted of VLA-alpha $a_{2}$ chain (GPIa) but still contained the normal amount of A-1A5-precipitated GPIc and approximately half of the normal content of beta chain GPIIa. These results indicate that the alloantibodies react with an epitope found only on the VLA-2 heterodimer (GPIa-IIa), and suggest that the cell donor E.A. may be heterozygous for coexpressed alloantibody-reactive and alloantibody unreactive forms of VLA-2.

The reactivity of the SLE alloantibody with only the GPIaGPIIa heterodimer and not other VLA forms suggested that the alloantigenic determinant might reside on the GPIa molecule. However, an alternative hypothesis was that the VLA-2 specific alloantigen was complex specific, perhaps residing on the GPIIa chain, but induced on GPIIa only by its specific association with GPIa and not other VLA alpha chains.

In an attempt to distinguish between these two possibilities, we studied the ability of the anti-VLA-2 alloantibody to react with separated VLA chains by the technique of Western blotting. Immunoisolated VLA molecules were subjected to either reduced or nonreduced SDS-PAGE, electrophoretically transferred to nitrocellulose, and then reacted with alloantibody-containing serum E35, or control serum. Under the conditions we employ for Western blotting, no reactivity of the alloantibody for any VLA chains was demonstrable (see Methods). In an attempt to increase the sensitivity of this assay we prepared platelet eluates enriched for E35 alloantibody. To prepare such eluates a large volume of E35 serum was incubated with platelets from an allotype positive normal donor, platelets washed, and then bound antibodies eluted under acid 


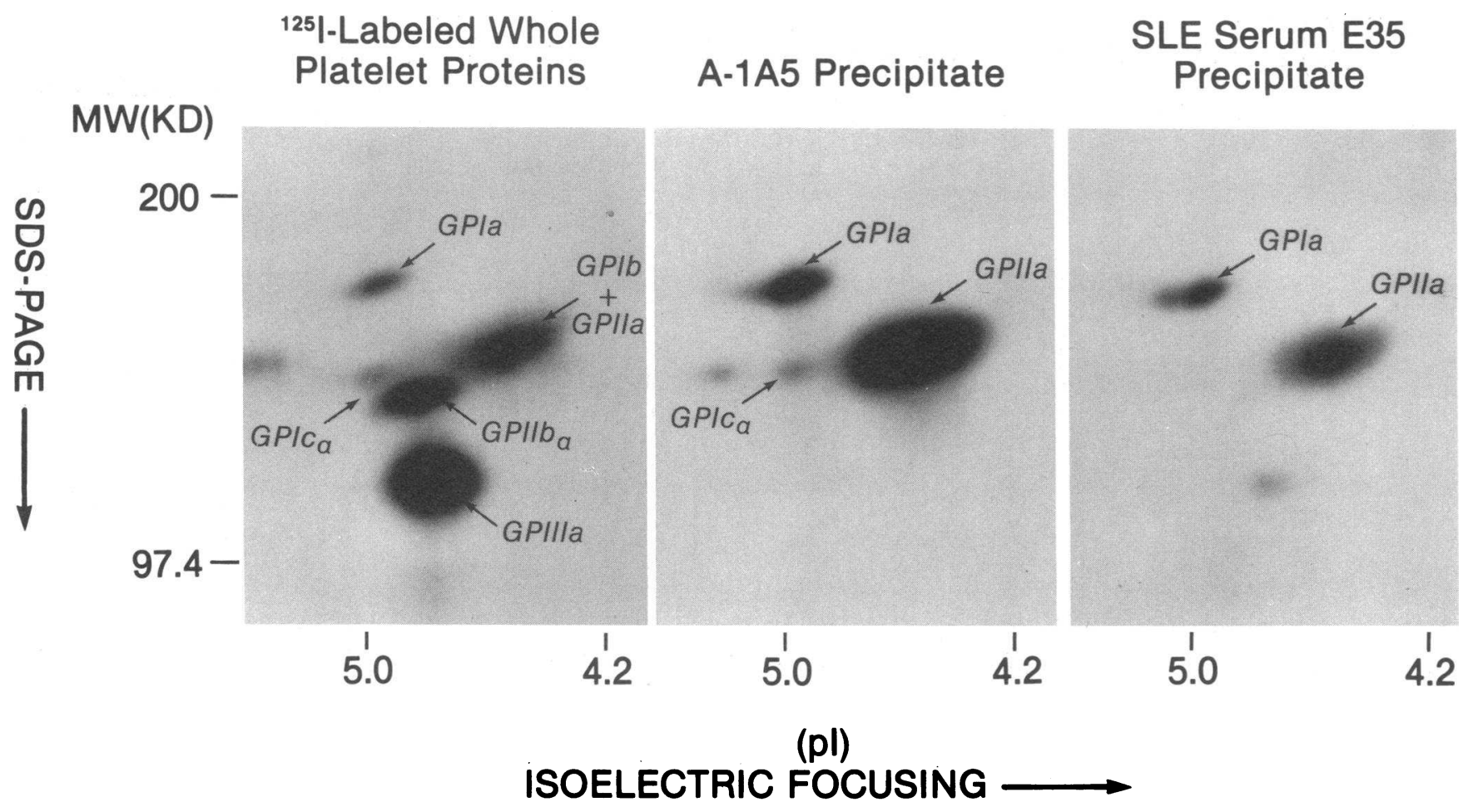

Figure 2. Comparison of the two-dimensional electrophoretic mobility of the platelet surface molecules precipitated by SLE serum E35 and monoclonal antibody A-1 A5. Immunoprecipitates were prepared from donor E.A.'s radiolabeled platelets and analyzed on two-dimensional O'Farrell gels with subsequent autoradiography as described in Methods. The migration positions of the components of the GPIIb/IIIa complex and platelet VLA molecules were determined by reference to our previously published work (12) and that of others (33). The SLE serum E35 precipitate contained two molecules which comigrated with A-1A5-precipitated GPIa and GPIIa. Furthermore, no GPIc-alpha was immunoprecipitated by this SLE serum, indicating that the serum specifically precipitates the VLA-2 but not other VLA heterodimers.

conditions. This eluate, which contained alloantibodies approximately fourfold concentrated over E35 sera, were tested for their ability to immunoblot to VLA antigens, and again demonstrated no reactivity with separated VLA chains.

Recent work suggests that platelet GPIa-GPIIa can func- tion as a platelet receptor for collagen $(20,21)$. If the GPIa-IIa heterodimer plays a role in collagen-induced platelet aggregation, and if anti-GPIa-IIa alloantibodies can bind to functionally significant regions of the GPIa-IIa molecule, then the alloantibodies might be found to interfere with collagen-induced

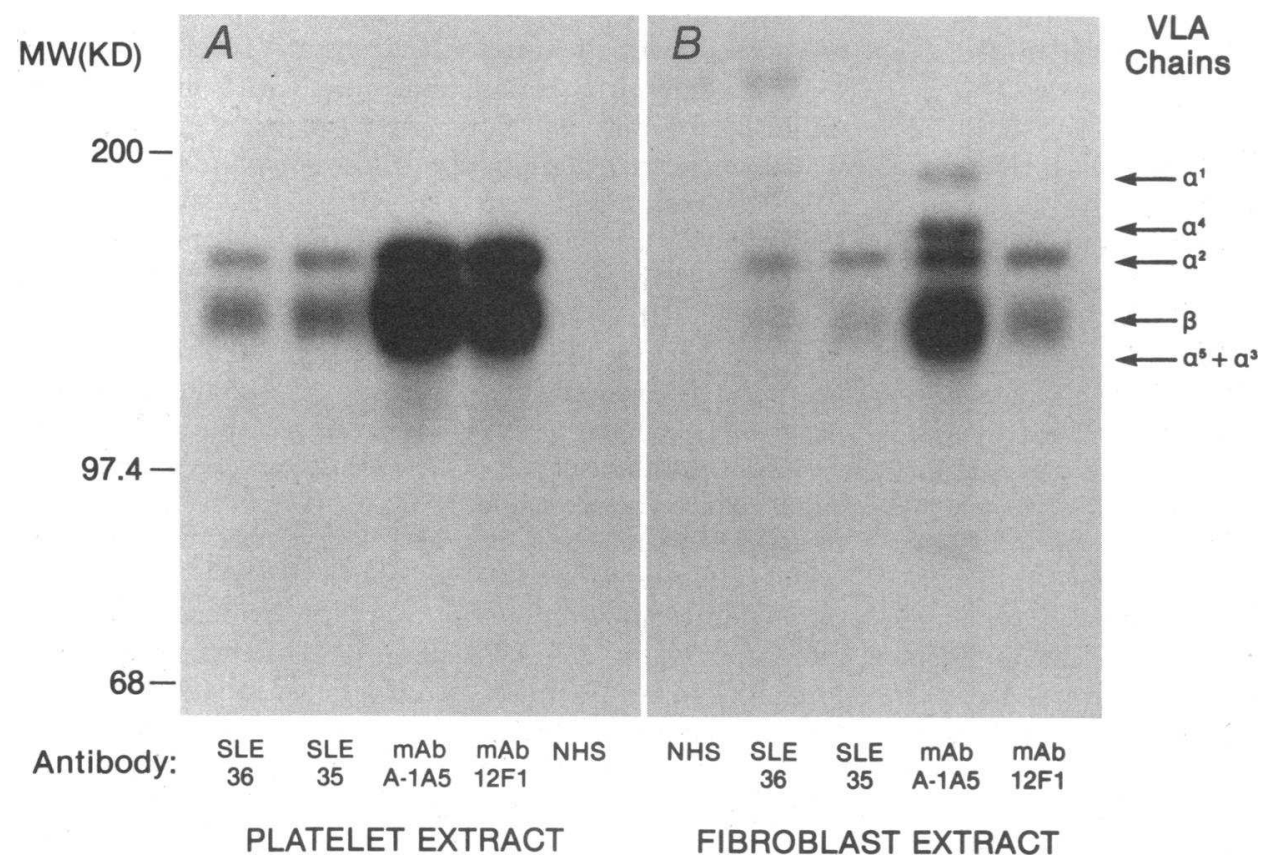

Figure 3. VLA-2 molecules on fibroblasts have the alloantigenic determinant found on platelets from the same donor. Platelets and skin fibroblasts obtained from donor R.W. were surface-radioiodinated, and then immunoprecipitated with SLE serum E36, E35, monoclonal antibody A-1A5 (specific for an epitope on the VLA-beta chain), monoclonal antibody $12 \mathrm{~F} 1$ (specific for an epitope on the VLA-alpha ${ }^{2}$ chain), or NHS. The precipitates were analyzed by reduced one-dimensional SDS-PAGE with autoradiography. The SLE alloantisera immunoprecipitated only material corresponding to the VLA-2

heterodimer (VLA-beta chain and alpha ${ }_{2}$ chain) from platelets $(A)$, and fibroblasts $(B)$ even though additional A-1A5 reactive VLA heterodimers were present in each extract. 


\section{PRECLEARING ANTIBODY:}

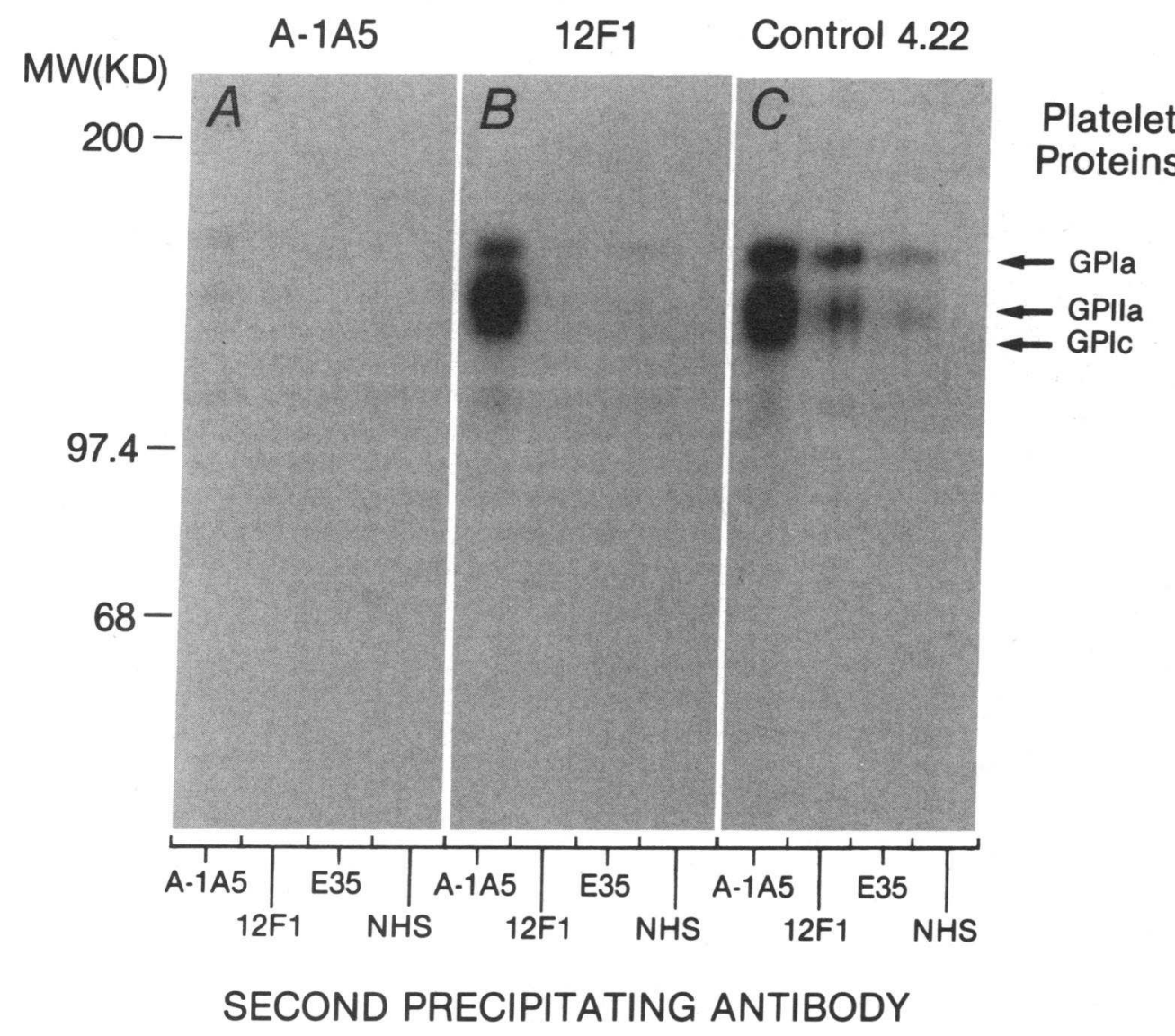

Figure 4. Preclearance of VLA-2 from platelet extracts by monoclonal antibody $12 \mathrm{~F} 1$ removes the molecules that react with the SLE alloantibodies. Radioiodinated platelets (donor E.A.) were incubated with SLE serum E35, washed, and solubilized in nonionic detergent. The extracts were then precleared with either A-1A5-Sepharose, 12F1-Sepharose, or control MAb 4.22-Sepharose as described in Methods. The resulting precleared extracts were then reacted with A-1A5 Sepharose or $12 \mathrm{~F} 1$ Sepharose to quantitate VLA molecules remaining in the extracts, and Protein A Sepharose to precipitate SLE serum E35-reactive molecules. Control MAb-precleared extracts $(C)$ contained $\mathrm{A}-1 \mathrm{~A} 5$ and $12 \mathrm{~F} 1$ reactive VLA molecules, as well as SLE serum E35-reactive GPIa and GPIIa. Control Protein A-Sepharose precipitates of extracts prepared from platelets preincubated with normal human serum contained no VLA molecules (far right lane in $A, B$, and $C$ ). A-1 A5-precleared extracts contained no residual A-1A5, 12F1 or E35 precipitable material. 12F1-precleared extracts were depleted of $12 \mathrm{~F} 1$ and $\mathrm{E} 35$ reactive material, but still contained A- 1 A5 reactive material indicating the presence of VLA molecules (GPIIa and GPIc) reactive with A-1A5, but unreactive with $12 \mathrm{~F} 1$. platelet aggregation. We examined the ability of alloantibodycontaining E35 plasma or alloantibody-enriched platelet eluate to interfere with ADP or collagen-induced aggregation of allotype bearing platelets, and found that the alloantibody did not interfere with, nor promote platelet shape change or aggregation.

\section{Discussion}

We have identified an antigenic polymorphism of human VLA molecules (now termed alloantigen $\mathrm{Hc}^{\mathrm{a}}$ ) by the alloreactivity of sera from two patients with SLE. These sera immunoprecipitated normal cell membrane glycoproteins that comigrated on two-dimensional O'Farrell gels with the components of the VLA-2 heterodimer, VLA-beta (GPIIa) and VLA-alpha ${ }_{2}$ (GPIa). The identity of the SLE sera-precipitated molecules with VLA-2 was confirmed by sequential immunoprecipitation experiments in which VLA-specific monoclonal antibodies and the SLE sera were found to react with the same proteins. The SLE antibodies reacted with the VLA proteins on the cells from some, but not all normal donors. The SLE serum-reactive allotype of VLA was present on cells from $23 \%$ of healthy human subjects. In the one case where it could be examined, the alloantigen was not present on cells from the SLE patient who provided the serum. Thus, the VLA reactivity in these SLE sera was alloreactive and not autoreactive. The two SLE sera with anti-VLA activity reacted concordantly towards a panel of normal donor cells, suggesting that both sera are reactive with the same allotypic determinant.

The antigenic site for the SLE alloantibodies appears to be located on the VLA-2 heterodimer. The alloantisera immunoprecipitated only the VLA-2 complex from extracts which also contain other VLA heterodimers. Additional glycoproteins should have been precipitated if the reactive epitope had been on the beta chain common to all VLA heterodimers. The structural differences between the SLE serum-reactive and unreactive allotypes of VLA molecules is fairly limited as it does not produce a detectable change in molecular weight or isoelectric point of VLA-alpha ${ }_{2}$ (GPIa). We performed experiments utilizing the technique of Western blotting in an attempt to sublocalize the alloantigenic determinant. In these experiments, alloantibodies were found to be unreactive with any VLA molecules, suggesting either that the alloantigen represents a complex-specific epitope created by the association of 
GPIa with GPIIa, or that the epitope resides on GPIa alone, but is denatured by the conditions employed for Western blot analysis.

This is the first description of an antigenic variation in human VLA molecules. A report of a patient with decreased/ absent amounts of platelet GPIa (VLA-alpha ${ }_{2}$ ) molecules has appeared (22). That defect is distinct from the alloantigenic differences in VLA molecules we have detected, since there is no decrease in the amount of GPIa on platelets from the SLE antibody-reactive and unreactive donors. Several platelet-specific alloantigen systems have been described including DUZO (23), Ko (24-26), $\mathrm{Pl}^{\mathrm{E}}$ (27), $\mathrm{Pl}^{\mathrm{A}}$ (= Zw) $(25,28,29)$, and Bak (30). Some of these diallelic systems have been localized to defined platelet glycoproteins. It is possible that the allelic system we have described on platelet GPIa may be identical with one of the as yet unassigned systems.

The clinical records of the two SLE patients in whom antiVLA alloantibodies were detected reveal a history of multiple pregnancies (and in one case transfusion) as the probable source of alloantigenic exposure. A history of multiple transfusions and/or pregnancy was found in most of our lupus patients yet only about $5 \%$ of them have made detectable amounts of VLA-reactive alloantibodies. It is therefore likely that these two SLE patients are homozygous for the alloantibody-unreactive allele of VLA-alpha ${ }_{2}$ (GPIa). We do not know if the unusual state of the SLE immune system enhances the response to alloimmunization with VLA-alpha $a_{2}$. None of our 21 healthy donors had anti-VLA reactivity, but they had little alloantigenic exposure.

The immunoprecipitation assay we have used to demonstrate an alloantigenic system involving VLA-2, is semiquantitative and is not capable of reliably discriminating between the homozygous and heterozygous state of an individual. The existence of an alloantigen system on GPIa-IIa will have been truly demonstrated only with the development of a more quantitative assay for the allotype which allows the performance of detailed genetic studies including family pedigrees. However, if we assume that the system we have identified is diallelic, and that the $77 \%$ of normal donors whose cells are unreactive with the SLE alloantibodies are homozygous for the alloantibody-unreactive allele, then the gene frequency of the

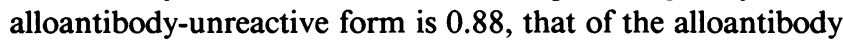
reactive form $\left(\mathrm{Hc}^{\mathrm{a}}\right)$ is $\mathbf{0 . 1 2}$, and homozygosity for the alloantibody reactive allele would be predicted to occur in $1.4 \%$ of normal individuals. This is approximately the same frequency of occurrence of homozygosity as is present for the $\mathrm{Pl}^{\mathrm{Al}}$-negative allele of platelet GPIIb/IIIa (2-3\%).

We have previously reported that when human VLA antigens are used to immunize rabbits and mice they behave as extremely potent immunogens (13). While alloantibodies directed at the $\mathrm{Pl}^{\mathrm{Al}}$ variant of GPIIb/IIIa are responsible for the majority of cases of both posttransfusion purpura and neonatal alloimmune thrombocytopenia $(14,31)$, these diseases can occur in the absence of evidence for GPIIb/IIIa alloimmunization. Given the potent immunogenicity of VLA antigens, it may be that alloantibodies directed at the variants of GPIa we have described may be responsible for some cases of posttransfusion purpura and/or neonatal purpura. Our immunoprecipitation assay utilizes staphylococcal Protein A to collect alloantibody-alloantigen immune complexes and therefore detects alloantibodies of the IgG class. IgM alloantibodies may be present in serum samples but remain undetected because of the weak binding of human IgM to Protein A under our experimental conditions.

Several lines of evidence suggest that the VLA-2 heterodimer may function as a cell surface receptor for collagen. The patient described by Nieuwenheis et al. who had a marked deficiency in platelet content of GPIa (and perhaps GPIIa) had a specific defect in platelet responsiveness to collagen (22). Furthermore, Santoro (32) has identified a 160,000 D platelet membrane protein that mediates the initial divalent cationdependent adhesion of platelets to collagen. Recent work indicates that this membrane protein is identical with platelet GPIa and that it binds to collagen as a GPIa-GPIIa complex $(20,21)$. The functional consequences of the structural variations that underlie the antigenic polymorphism of GPIa described in this report are unknown.

\section{Acknowledgments}

We thank Martin Hemler for provision of monoclonal A-1A5 and for helpful discussions.

These studies were supported by a grant from the state of California through the Universitywide Task Force on AIDS (V. L. Woods, Jr.) and by National Institutes of Health grants HL-30480, AM-30036, and AI-24624. K. D. Pischel is the recipient of an Arthritis Foundation Investigator Award.

Note added in proof. Recent experiments indicate that the $\mathrm{Hc}^{\mathrm{a}}$ antigen described in this report is identical with the alloantigen $\mathrm{Zav}^{\mathrm{a}}$ and the alloantigen $\mathrm{Br}^{\mathrm{a}}$.

\section{References}

1. Hemler, M. E., C. F. Ware, and J. L. Strominger. 1983. Characterization of a novel differentiation antigen complex recognized by a monoclonal antibody (A-1A5): unique activation-specific molecular forms on stimulated T cells. J. Immunol. 131:334-340.

2. Hemler, M. E., F. Sanchez-Madrid, T. J. Flotte, A. M. Krensky, S. J. Burakoff, A. K. Bhan, T. A. Springer, and J. L. Strominger. 1984. Glycoproteins of 210,000 and $130,000, M$. W. on activated T cells. Cell distribution and antigenic relation to components on resting cells and T cell lines. J. Immunol. 132:3011-3018.

3. Hemler, M. E., J. G. Jacobson, M. B. Brenner, D. Mann, and J. S. Strominger. 1985. VLA-1: a T cell surface antigen which defines a novel late stage of human T cell activation. Eur. J. Immunol. 15:502508.

4. Hemler, M. E., J. G. Jacobson, and J. L. Strominger. 1986. Biochemical characterization of VLA-1 and VLA-2. Cell surface heterodimers on activated T cells. J. Biol. Chem. 260:15246-15252.

5. Hemler, M. E., C. Huang, and L. Schwarz. 1987. The VLA protein family. Characterization of five distinct cell surface heterodimers each with a common $130,000 \mathrm{Mr}$ beta subunit. J. Biol. Chem. 262:3300-3309.

6. Hemler, M. E., C. Crouse, Y. Takada, and A. Sonnenberg. 1988. Multiple very late antigen (VLA) heterodimers on platelets. J. Biol. Chem. 263:7660-7665.

7. Takada, Y., C. Huang, and M. E. Hemler. 1987. Fibronectin receptor structures are included in the VLA family of heterodimers. Nature (Lond.). 326:607-609.

8. Hynes, R. O. 1987. Integrins: A family of cell surface receptors. J. Cell. 48:549-554.

9. Pytela, R., M. D. Pierschbacher, M. H. Ginsberg, E. F. Plow, and E. Ruoslahti. 1986. Platelet membrane glycoprotein IIb/IIIa: Member of a family of arg-gly-asp-specific adhesion receptors. Science (Wash. DC). 231:1559-1562. 
10. Takada, Y., J. L. Strominger, and M. E. Hemler. 1987. The very late antigen family of heterodimers is part of a superfamily of molecules involved in adhesion and embryogenesis. Proc. Natl. Acad. Sci. USA. 84:3239-3243.

11. Pischel, K. D., H. G. Bluestein, and V. L. Woods. 1985. Lymphocytes bear molecules (VLA) that are antigenically and structurally similar to GPIa, GPIc, and GPIIa. Blood. 66:310a. (Abstr.)

12. Pischel, K. D., H. G. Bluestein, and V. L. Woods. 1988. Platelet glycoproteins Ia, Ic, and IIa are physicochemically indistinguishable from the VLA antigens adhesion-related proteins of lymphocytes and other cell types. J. Clin. Invest. 81:505-513.

13. Pischel, K. D., H. G. Bluestein, and V. L. Woods. 1986. Very late activation antigens are human leukocyte-neuronal cross-reactive cell surface antigens. J. Exp. Med. 164:393-406.

14. Pfueller-Sharron, L. 1985. Immunology of the platelet surface. In Platelet Membrane Glycoproteins. J. N. George, A. T. Nurden, and D. R. Phillips, editors. Plenum Press, New York. 331-344.

15. Woods, V. L., E. H. Oh, D. Mason, and R. McMillan. 1984. Autoantibodies against the platelet glycoprotein IIb/IIIa complex in patients with chronic ITP. Blood. 63:368-375.

16. Laubscher, A., H. G. Bluestein, S. A. Spector, and N. J. Zvaifler. 1988. Generation of human cytomegalovirus-specific cytotoxic T-lymphocytes in a short-term culture. J. Immunol. Methods. 110:69-77.

17. Pischel, K. D., S. D. Marlin, T. A. Springer, V. L. Woods, and H. G. Bluestein. 1987. Polymorphism of lymphocyte function-associated antigen-1 demonstrated by a lupus patient's alloantiserum. $J$. Clin. Invest. 79:1607-1614.

18. Pischel, K. D., M. E. Hemler, C. Huang, H. G. Bluestein, and V. L. Woods. 1987. Use of the monoclonal antibody $12 \mathrm{~F} 1$ to characterize the differentiation antigen VLA-2. J. Immunol. 138:226-233.

19. Shulman, N. R., V. J. Marder, M. C. Hiller, and E. M. Collier. 1964. Platelet and leukocyte isoantigens and their antibodies. Serologic, physiologic, and clinical studies. Prog. Hematol. 4:222-304.

20. Santoro, S. A., S. M. Rajpara, W. D. Staatz, and V. L. Woods. 1988. Isolation and characterization of a platelet surface collagen binding complex related to VLA-2. Biochem. Biophys. Res. Commun. 153:217-223.

21. Kunicki, T. J., D. J. Nugent, S. J. Staats, R. P. Orchekowski, E. A. Wagner, and W. G. Carter. 1988. The human fibroblast class II extracellular matrix receptor mediates platelet adhesion to collagen and is identical to the platelet glycoprotein Ia-IIa complex. J. Biol. Chem. 263:4516-4519.

22. Nieuwenheis, H. K., J. W. Akkerman, W. P. Houdijk, and J. J. Sixma. 1985. Human blood platelets showing no response to collagen fail to express surface glycoprotein Ia. Nature (Lond.). 318:470-472.

23. Moulinier, J. 1957. Iso-immunisation maternelle anti-plaquettaire et purpura neonate. In European Society of Haematology. S. Karger, Basel. 817-820.

24. Van der Weerdt, C. M., H. Van de Wiel-Dorfmeyer, C. P. Engelfriet, and J. J. Van Loghem. 1961. A new platelet antigen. In European Society of Haematology. S. Karger, Basel. 379-382.

25. Van der Weerdt, C. M., L. E. Veenhoven-Von Reisz, and J. J. Van Loghem. 1963. The $\mathrm{Zw}$ blood group system in platelets. Vox Sang. 8:513-530.

26. Dausset, J., and P. Berg. 1963. Un nouvel example d'anticorps antiplaquettaire Ko. Vox Sang. 8:341-347.

27. Shulman, N. R., V. J. Marder, M. C. Hiller, and E. M. Collier. 1964. Platelet and leukocyte isoantigens and their antibodies: serologic, physiologic, and clinical studies. Prog. Haematol. 4:222-304.

28. Van Loghem, J. J., H. Dorfmeyer, M. van der Hart, and F. Schreuder. 1959. Serological and genetical studies on a platelet antigen (Zw). Vox Sang. 4:161-169.

29. Shulman, N. R., R. H. Aster, A. Leitner, and M. C. Hiller. 1961. Immunoreactions involving platelets. V. Post-transfusion purpura due to a complement-fixing antibody against a genetically controlled platelet antigen. J. Clin. Invest. 40:1597-1620.

30. Von dem Borne, A. E. G. K., E. von Riesz, F. W. A. Verheugt, J. W. ten Cate, J. G. Koppe, C. P. Engelfriet, and L. E. Nijenhuis. 1980. Bak, a new platelet-specific antigen involved in neonatal alloimmune thrombocytopenia. Vox Sang. 39:113-120.

31. Pegels, J. G., E. C. E. Bruynes, C. P. Engelfriet, and A. E. G. K. von dem Borne. 1981. Post-transfusion purpura: a serological and immunochemical study. Br. J. Haematol. 49:521-530.

32. Santoro, S. A. 1986. Identification of a 160,000 platelet membrane protein that mediates the initial divalent cation-dependent adhesion of platelets to collagen. Cell. 46:913-920.

33. Clemetson, K. J., A. Capitanio, and E. F. Luscher. 1979. High resolution two-dimensional electrophoresis of the proteins and glycoproteins of human blood platelets and platelet membrane. Biochim. Biophys. Acta. 553:11-24. 